\title{
The CFHTLS strong lensing legacy survey
}

\section{Survey overview and T0002 release sample ${ }^{\star}$}

\author{
R. A. Cabanac ${ }^{1}$, C. Alard ${ }^{2}$, M. Dantel-Fort ${ }^{2}$, B. Fort ${ }^{3}$, R. Gavazzi ${ }^{4}$, P. Gomez ${ }^{5}$, J. P. Kneib ${ }^{6}$,
}

O. Le Fèvre ${ }^{6}$, Y. Mellier ${ }^{3,2}$, R. Pello ${ }^{4}$, G. Soucail ${ }^{4}$, J. F. Sygnet ${ }^{3}$, and D. Valls-Gabaud ${ }^{1,4,7}$

${ }^{1}$ Canada-France-Hawaii Telescope, 65-1238 Mamalahoa Hw., Kamuela, Hawaii 96743, USA

e-mail: cabanac@cfht.hawaii.edu

2 Observatoire de Paris, LERMA, CNRS-UMR8112, 61 avenue de l'Observatoire, 75014 Paris, France

3 Institut d'Astrophysique de Paris, CNRS-UMR7095 and Université Pierre et Marie Curie, 98bis boulevard Arago, 75014 Paris, France

4 Laboratoire d'Astrophysique de Toulouse-Tarbes, CNRS-UMR5572 and Université Paul Sabatier Toulouse III, 14 avenue Édouard Belin, 31400 Toulouse, France

5 Gemini Observatory Northern Operations Center, 670 N. A'ohoku Place, Hilo, Hawaii 96720, USA

${ }^{6}$ Laboratoire d'Astrophysique de Marseille, traverse du Siphon, BP 8, 13376 Marseille Cedex 12, France

7 Observatoire de Paris, GEPI, CNRS-UMR8111, 5 place Jules Janssen, 92195 Meudon Cedex, France

Received 12 June 2006 / Accepted 5 September 2006

\section{ABSTRACT}

\begin{abstract}
Aims. We present data from the CFHTLS Strong Lensing Legacy Survey (SL2S). Due to the unsurpassed combined depth, area and image quality of the Canada-France-Hawaii Legacy Survey it is becoming possible to uncover a large, statistically well-defined sample of strong gravitational lenses which spans the dark halo mass spectrum predicted by the concordance model from galaxy to cluster haloes.

Methods. We describe the development of several automated procedures to find strong lenses of various mass regimes in CFHTLS images.

Results. The preliminary sample of about 40 strong lensing candidates discovered in the CFHTLS T0002 release, covering an effective field of view of $28 \mathrm{deg}^{2}$ is presented. These strong lensing systems were discovered using an automated search and consist mainly of gravitational arc systems with splitting angles between 2 and 15 arcsec. This sample shows for the first time that it is possible to uncover a large population of strong lenses from galaxy groups with typical halo masses of about $10^{13} h^{-1} M_{\odot}$. We discuss the future evolution of the SL2S project and its main scientific aims for the next 3 years, in particular our observational strategy to extract the hundreds of gravitational rings also present in these fields.
\end{abstract}

Key words. gravitational lensing - surveys - cosmology: dark matter - galaxies: fundamental parameters - galaxies: clusters: general - galaxies: halos

\section{Introduction}

The observation of gravitational lensing effects produced by mass concentrations is a powerful tool to directly probe dark matter haloes and their interplay with visible mass (Schneider et al. 1992; Blandford \& Narayan 1992; Miralda-Escude \& Lehar 1992; Mellier 2002; Kneib et al. 2003). Both strong and weak lensing regimes are therefore widely used to explore the dark matter distribution properties, either from observations of individual cases (Warren et al. 1996; Cabanac et al. 2005; Willis et al. 2006) or from statistical analyses of large samples of lensed galaxies or quasars (for a comprehensive review Mellier \& Meylan 2005).

\footnotetext{
* Based on observations obtained with MegaPrime/MegaCam, a joint project of CFHT and CEA/DAPNIA, at the Canada-France-Hawaii Telescope (CFHT) which is operated by the National Research Council (NRC) of Canada, the Institut National des Sciences de l'Univers of the Centre National de la Recherche Scientifique (CNRS) of France, and the University of Hawaii. This work is based in part on data products produced at TERAPIX and the Canadian Astronomy Data Centre as part of the Canada-France-Hawaii Telescope Legacy Survey, a collaborative project of $\mathrm{NRC}$ and CNRS.
}

On galaxy scales, ongoing lens surveys are now providing reliable descriptions of galaxies and a clearer understanding of the key issues regarding the star and dark matter distributions. As pointed out by Rusin et al. (2003) and Rusin \& Kochanek (2005), using a large sample of well-studied strong lenses, it is possible to describe galaxy structure and the transition between the inner stellar matter-dominated and the outer dark matter-dominated galaxy haloes, without being sensitive to the mass-sheet degeneracy. It complements the galaxy-galaxy lensing methods that explore galaxy haloes on much larger scales (Hoekstra et al. 2004; Seljak et al. 2005). Detailed lens studies have led to an observational technique based on spectroscopic selection of compact lensing galaxy candidates (Hall et al. 2000; Hewett et al. 2000) that was fully exploited by Bolton et al. (2006); Treu et al. (2005); Koopmans et al. (2006) and Willis et al. (2006). Using the large spectroscopic data base of the SDSS, Bolton et al. (2006) have identified and studied a first set of 20 rings (which are merged compact multiple arc systems) among a total sample of 120 candidates. The first analysis of this Sloan Lens ACS (SLACS) data has provided a better description of the structural parameters of isolated galaxy lenses at low 
redshift than multiply-imaged quasars (Treu et al. 2005; Koopmans et al. 2006). Moreover, their study of the fundamental plane of E/SO galaxies improves on ealier work (Kochanek et al. 2000) by combining a standard dynamical analysis with the strong lens constraints. This allows them to break the degeneracy between anisotropy of the stellar velocity tensor and the lens gravitational potential (for a similar approach Miralda-Escude 1995). They also showed that the lens galaxies of the SLACS sample nicely follow the E/S0 fundamental plane, being only slightly skewed towards the more massive objects. However, the SLACS is limited to small rings $\left(<3^{\prime \prime}\right)$ by the aperture of the spectrograph fibers and to nearby lenses $\left(z_{\max }(\right.$ lens $)<0.5$, $z_{\max }$ (source) $<0.8$; cutoff of the SDSS spectroscopic follow-up, and SLACS selection criteria). An extension of the method to larger redshifts should enlarge the sample significantly and will benefit from the higher efficiency of strong lensing at redshift $\sim 0.5$ for galaxy sources at redshift above 1 . Such ongoing efforts by Willis et al. (2006) push $z_{\max }$ (source) $<1.3$.

Because clusters of galaxies are more complex systems than galaxies alone, it is not yet clear to what extend systematics, projection and selection effects hamper a reliable description of cluster size haloes. While the number of cluster-size haloes can in principle be derived from weak lensing studies (Hetterscheidt et al. 2005), the detailled description of the cluster halo structures and of their light versus mass distribution properties is still uncertain. Ground-based+HST observations and strong+weak lensing analyses of individual or samples of clusters of galaxies seem to indicate that more complex radial profiles (NFW like or power law with a flat core) than singular isothermal are required to fit the lensing data (Kneib et al. 2003; Gavazzi et al. 2003; Broadhurst et al. 2005). However for giant arcs in clusters of galaxies, large optically selected samples of strong lensing groups of galaxies are not available yet. The Red-sequence Cluster Survey (Gladders et al. 2003) is a first attempt to systematically find strong lensing around clusters and groups of galaxies. But their relatively low detection sensitivity has lead to the discovery of only eight cluster-like structures at $z>0.64$ over a 90 sq. degree field.

A key issue is to understand the transition between galaxyscale to cluster-scale halo structures. Quasar lenses and gravitational arcs have mostly probed two regimes of halo masses: galaxies and clusters of galaxies, but bring only weak constraints on the intermediate mass range $\left(10^{12}-10^{14} M_{\odot}\right)$ which is important for the assembly of large scale structures (Kochanek et al. 2001; Grant et al. 2004; Fassnacht et al. 2005; Mathews et al. 2005; Oguri 2006). The study of groups of galaxies in the CNOC survey using weak lensing by Parker et al. (2005) yielded the first constraints of their averaged mass-to-light ratios but nothing on their inner structures or on whether groups are self-similar.

In summary, no homogeneous sample of strong lenses have been built so far that covers the full dark matter halo mass spectrum because of the lack of a large, deep sky survey with a subarc-second seeing. We will demonstrate here that with the CFHT Legacy Survey (CFHTLS), due to its combined depth, area and image quality of the data, we are able to find strong lensing systems around a wide mass spectrum of structures. Indeed, the three $7 \times 7-\mathrm{deg}^{2}$ wide patches together with the four $1 \times 1-\mathrm{deg}^{2}$ deep patches of CFHTLS allow us to build up a large sample of strong galaxy-, group-, and cluster-scale lenses with a welldefined selection function and sampling variance, as well as to explore halo properties at different depths and redshifts.

In order to do this, a set automated procedures has been developed to detect various types of strong lensing events. It has been successfully tested on the T002 release. We present in this paper a preliminary sample built with these selection procedures. Although these procedures are not yet fully optimized, we can nevertheless uncover within the CFHTLS a large population of "group lenses", a new class of lenses with multiple image separation of 2 to 7 ". We also show that it is possible to implement a dedicated procedure to recover gravitational rings with Einstein radii below $2^{\prime \prime}$. The CFHTLS Strong Lensing Legacy Survey (SL2S) should allow us to extract the whole lensing mass spectrum from galaxies to clusters of galaxies, in a homogeneous and statistically well-defined procedure.

The paper is organized as follow: Sect. 2 summarizes the present state of the CFHTLS and the data used in this preliminary study. Section 3 presents the SL2S project itself with a brief discussion of the automated search procedures, as well as the first results obtained for group lenses. A full description of the selection procedures will be provided elsewhere. Sections 4 and 5 discuss the future of the project.

Throughout the paper we use a flat $\Lambda \mathrm{CDM}$ cosmology $\left(\Omega_{\mathrm{m}}=0.3, \Omega_{\Lambda}=0.7\right)$, all observables are computed with $H_{0}=70 \mathrm{hm} \mathrm{s}^{-1} \mathrm{Mpc}^{-1}$ and magnitudes are given in the $A B$ system unless specified otherwise.

\section{The Canada-France-Hawaii telescope legacy survey}

\subsection{Description}

The Canada-France-Hawaii Telescope Legacy Survey (CFTHLS) is a major photometric survey of more than 450 nights over 5 years (started on June 1st, 2003) using the wide field imager MegaPrime which covers $\sim 1$ square degree on the sky, with a pixel size of $0.186^{\prime \prime}$. The project is comprehensively described in http://www.cfht.hawaii.edu/ Science/CFHLS/ and links therein. The CFHTLS has two components aimed at extragalactic studies: a very Deep component made of 4 pencil-beam fields of $1 \mathrm{deg}^{2}$ and a Wide component made of 3 mosaics covering $170 \mathrm{deg}^{2}$ in total, both in 5 broadband filters. The data are pre-reduced at CFHT with the Elixir pipeline ${ }^{1}$ which removes the instrumental artefacts in individual exposures. The CFHTLS images are then evaluated, astrometrically calibrated, photometrically inter-calibrated, resampled and stacked by the Terapix group at the Institut d'Astrophysique de Paris (IAP) and finally archived at the Canadian Astronomy Data Centre (CADC). Terapix also provides weightmap images, quality assessements meta-data for each stack as well as mask files that mask straylight, saturated stars and defects on each image. The preliminary SL2S sample presented here is based on the T0002 release (July 2005), corresponding to data obtained between June 1st, 2003 and Nov. 22nd, 2004. A detailed description of this release is given at the Terapix web site http://terapix.iap.fr and the Terapix T0002 release document (Mellier et al 2005: http: //terapix . iap. fr/IMG/pdf/Newterapixdoc . pdf). The T0002 release includes 40 stacked fields in the Wide survey observed in broadband $g^{\prime}, r^{\prime}$ and $i^{\prime}$ filters, and a stack of the 4 Deep fields in the 5 bands, for a total area of $44 \mathrm{deg}^{2}$, or ca. $28 \mathrm{deg}^{2}$ of unmasked area, i.e. area not contaminated by instrumental artefacts from bright stars (internal reflections, bleeding). Table 1 summarizes the main characteristics of the data used in the paper. The 4 Deep fields are much deeper, with an average seeing ranging from $1.0^{\prime \prime}$ in $u^{*}$ to $0.85^{\prime \prime}$ in $z^{\prime}$. The Wide survey is presently available in 3 filters only with an

\footnotetext{
${ }^{1}$ http://www. cfht .hawaii.edu/Instruments/Elixir/
} 
Table 1. CFHTLS: Terapix T0002 release (July 2005).

\begin{tabular}{lccccc}
\hline \hline \multirow{2}{*}{ Deep fields } & \multicolumn{5}{c}{ Magnitudes Limits } \\
& $u^{*}$ & $g^{\prime}$ & $r^{\prime}$ & $i^{\prime}$ & $z^{\prime}$ \\
\hline D1 & 26.4 & 26.3 & 26.1 & 25.9 & 24.9 \\
D2 & 26.1 & 25.8 & 25.8 & 25.4 & 24.3 \\
D3 & 25.8 & 26.3 & 26.3 & 25.9 & 24.7 \\
D4 & 26.2 & 26.3 & 26.3 & 25.7 & 24.9 \\
\hline
\end{tabular}

Wide fields

Average Magnitudes Limits ${ }^{1}$

$\begin{array}{llllll}\text { W1 } & - & 25.5 & 24.5 & 24.5 & - \\ \text { W2 } & - & 25.4 & 24.7 & 24.6 & - \\ \text { W3 } & - & 25.7 & 25.0 & 24.6 & - \\ \text { W1 area (unmasked) } & & & 20 & (11.4) \mathrm{deg}^{2} & \\ \text { W2 area (unmasked) } & & 8 & (5.0) \mathrm{deg}^{2} & \\ \text { W3 area (unmasked) } & & & & (9.0) \mathrm{deg}^{2} & \end{array}$

${ }^{1} 50 \%$ completeness limit in $A B$ mag $\left(A B\right.$ to Vega $u^{*}-0.35, g^{\prime}+0.09$, $\left.r^{\prime}-0.17, i^{\prime}-0.40, z^{\prime}-0.55\right)$.

average seeing of $1.0^{\prime \prime}$ in $g^{\prime}$ and $0.9^{\prime \prime}$ in $r^{\prime}$ and $i^{\prime}$. But not surprisingly, the Wide survey is more suited to our strong lensing selection processes, because of its wide angular coverage.

\subsection{Strong lensing number predictions}

In this paper we call a ring any compact system of arcs where multiple images merge into a single ring-like image surrounding the deflector; all other types of multiple systems are either called (giant) arcs or arclets. The previous observations of QSO lenses and the results from the most recent simulations (lensing optical depths) give good estimates of number densities of lenses associated at various deflecting halo masses (i.e. for splitting angles) (Oguri 2006, Figs. 9 and 10, and Table 1). Typically, for any giant arc detected in a cluster, we expect about 4 times more arc(et)s systems in haloes corresponding to group masses, and 20 times more gravitational rings associated with lens galaxy haloes (assuming equivalent detection limit and angular magnification factor in all cases). We will see below that the preliminary number of group-lenses, based on the first (beta) version of the arc detection software, is found to be $\sim 0.5 \mathrm{deg}^{-2}$ in the CFHTLS data. Extrapolating Oguri's distribution to the total area of the CFHTLS Wide component $\left(170 \mathrm{deg}^{2}\right)$ yields $\sim 75$ group arcs, $\sim 400$ galaxy rings, and $\sim 20$ cluster arcs. The number of lenses will be very small per Megacam field whatever the deflector mass regime (cluster, group or galaxy). Their detection is therefore very challenging as the lensed features are hidden within a huge number of faint background galaxies. The only way to find them efficiently is to use well-defined automated procedures (e.g. see Lenzen et al. 2004). In practice such an approach has already been proposed for some large numerical simulations of lensing configurations, mostly in simulated samples of clusters of galaxies (Horesh et al. 2005).

\section{Automated software for strong lensing candidate detection}

One of the goals of the SL2S is to provide a complete and homogeneous sample of strong lenses with known detection efficiency with regard to a series of observational parameters. This goal requires us not only to develop a comprehensive series of simulations taking into account the most common biases such as seeing, PSF variation, crowding, limiting magnitude, surface brightness, and arc length/radius/thickness distribution, but also requires us to automate the detection procedures. We are thus currently developing three complementary algorithms covering various regimes of strong lensing. The first one is a gravitational $A R C$ detector (mostly for groups and clusters). The second is a detector of compact RING candidates (which will then require either spectroscopic or higher resolution images for confirmations). The third one is a MULTIPLET detector, aimed at detecting peculiar and rare multiple arclet systems that cannot be recognized by the $A R C$ detector. This includes badly resolved multiple image systems or configurations which may form in potential saddles. The software selects multiple image configurations based only on generic properties like color, distance, flux ratio and shear orientation, if any, and the geometric distribution of multiple images. However we have not yet been able to optimize this procedure on ground-based images so the MULTIPLET detector will not be addressed further.

\section{1. $A R C$ detector}

Giant arcs are in principle the most straightforward images to identify and detect through direct pattern recognition (Lenzen et al. 2004; Seidel \& Bartelmann 2006). They are known to occur around massive clusters $\left(>10^{14} h^{-1} M_{\odot}\right)$ and show radii up to $\sim 40^{\prime \prime}$ in very luminous X-ray clusters (see for example the spectacular case of A 1689 Broadhurst et al. 2005). When arcs appear around groups of galaxies, their radii are five to ten times smaller and the seeing makes them more difficult to identify from ground-based imaging. A full description of the technique overcoming this difficulty is given in Alard (2006). Briefly, the arc-detector algorithm detects elongated structures and analyzes the local properties of these structures. Elongated objects are defined as very narrow objects along one direction with a width nearly equal to the size of the seeing. We model the elongated arc(let)s structures as small rectilinear objects having the width of the seeing along one direction, and 3 times this width in the other direction. This condition sets the detection threshold below an Einstein radius of $3^{\prime \prime}$ for an arc angular aperture of about $60^{\circ}$. Each object in the image is decomposed in a series of contiguous elements aligned along the tangential direction of the elongated structure. The full object is re-constructed by associating the areas covered by the different elements. Once a set of pixels is associated with the object, we compute its general properties, size, color, curvature, etc. To ease the pattern recognition the routine also requires both the $g^{\prime}-i^{\prime}$ color and surface brightness of the elongated objects to be constant. Finally we produce a catalogue of candidates and a set of associated color images for visual inspection according to a selected set of parameters that fully describes the detection procedure.

The $A R C$ detector is very efficient at detecting extremely faint arcs over a large range of splitting angles and will be used in subsequent CFHTLS releases (cf. Table 2).

\subsection{RING detector}

The ring detector is aimed at detecting compact rings around centers of isolated galaxies $\left(<10^{13} h^{-1} M_{\odot}\right)$. Most of the ring radii are in the range $0.5-2.0^{\prime \prime}$ and rings are usually hidden within the deflector. Seeing and intrinsic galaxy morphologies, like dust lanes and face-on spirals, make the ring detection challenging. We use an "object-oriented" routine, RING, that will be described in a future paper (Gavazzi et al., in preparation). Presently, we focus on the 4 Deep fields that have been observed in 5 filters and for which photometric redshift catalogs 


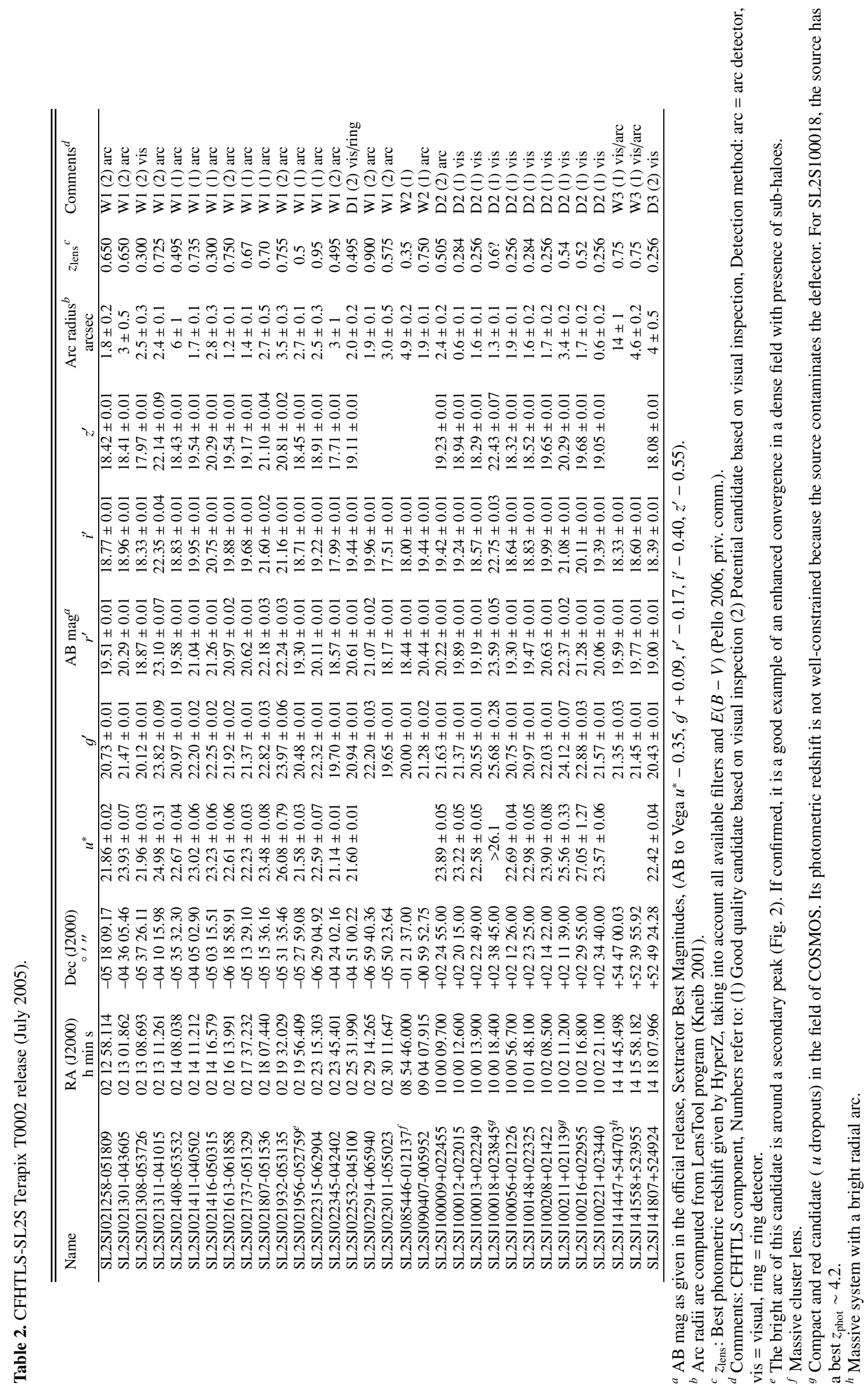




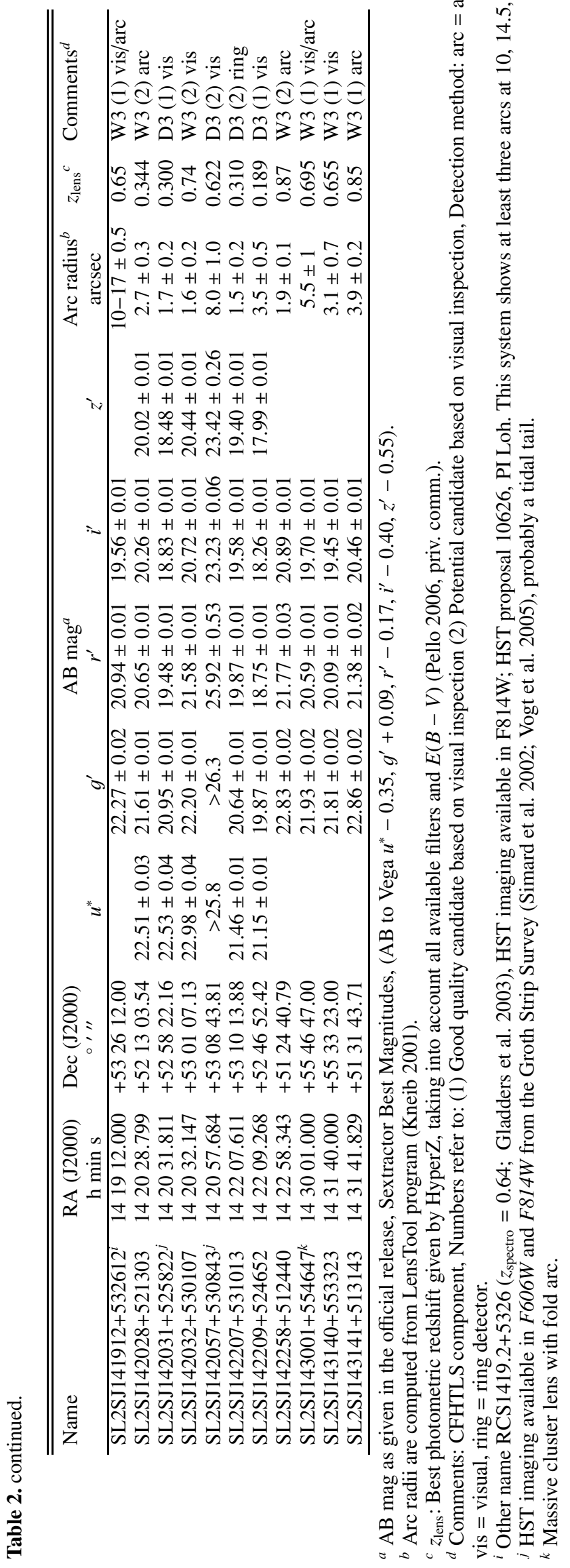

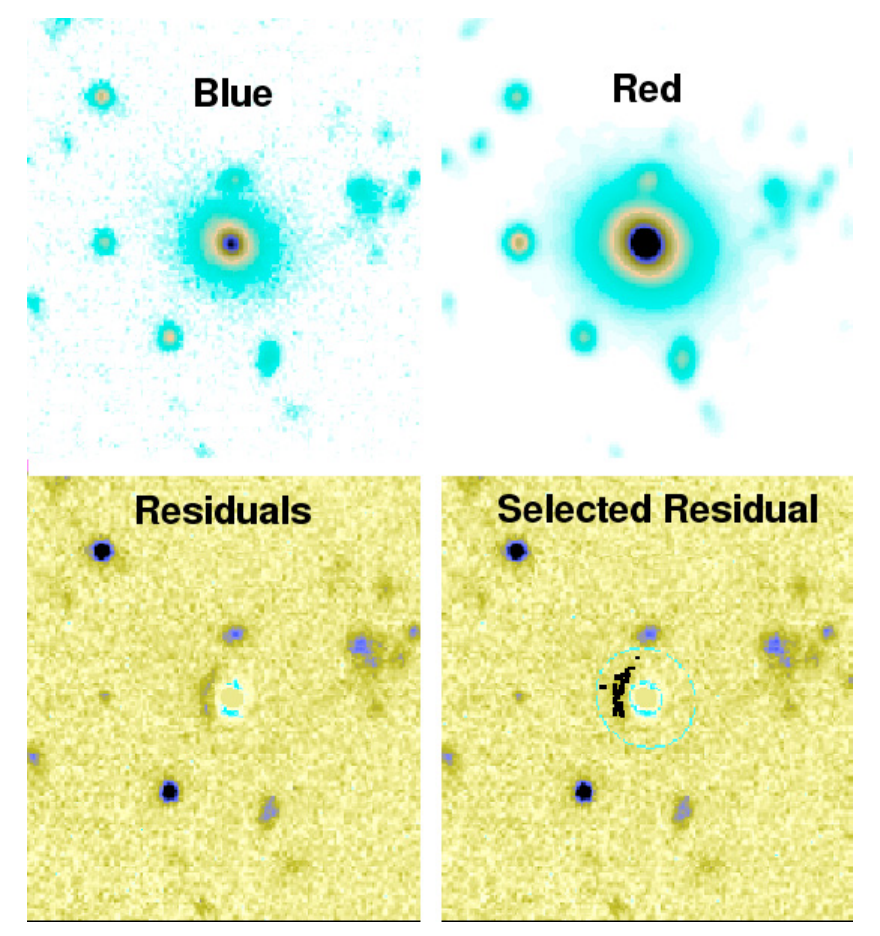

Fig. 1. Schematics of the ring detection procedure (here SL2SJ100013+022249). The candidates are selected from CFHTLS ellipticals (top). $g^{\prime}-i^{\prime}$ color images reveal strong residual signal (bottom).

are built. These catalogs include a photometric redshift estimate, the best fit spectral type and information on the absolute magnitudes in different spectral bands. Selecting all objects catalogued as E/S0 galaxies, the routine filters out the large scale light distribution of the deflectors, using $g^{\prime}-\alpha \times i^{\prime}$ color vignettes. When the profile of the potential deflector does not depend much on color, or when the deflector profile is smooth on large scales, the deflector is subtracted cleanly and any residual comes from a superimposed smaller-scale anisotropy. The routine selects the lens candidates based on the computed residuals above sky noise in the range $0.8-2.5^{\prime \prime}$ (Fig. 1). We are currently optimizing the method with a sample of 10 lens candidates common to the COSMOS field and the CFHTLS-D2 field. The first results are encouraging: most of the rings with radii larger than $0.8^{\prime \prime}$ seem to be recovered. However the method has two limitations. First, any ring candidate smaller than the seeing radius is lost. Second, the procedure is a good filtering method, efficient in removing massive ellipticals that are not lenses, but still produces false candidates among S0 galaxies. Therefore a final eyeball selection is currently still required to select good candidates.

The resulting candidates will need confirmation by spectroscopic identification of both the redshift of the lens and the lensed galaxy. In addition, high-resolution spectroscopy will help determine to the lens stellar velocity dispersion, and higher resolution images, using for example the Hubble Space Telescope, will allow us to address the lens modelling with high accuracy. Thus, the method is mostly able to select, among a large number of massive ellipticals, a small sample of good strong lensing candidates.

\subsection{Lens modelling}

A large number of algorithms of gravitational lens modelling are available (e.g. Kneib et al. 1993; Saha \& Williams 1997; 


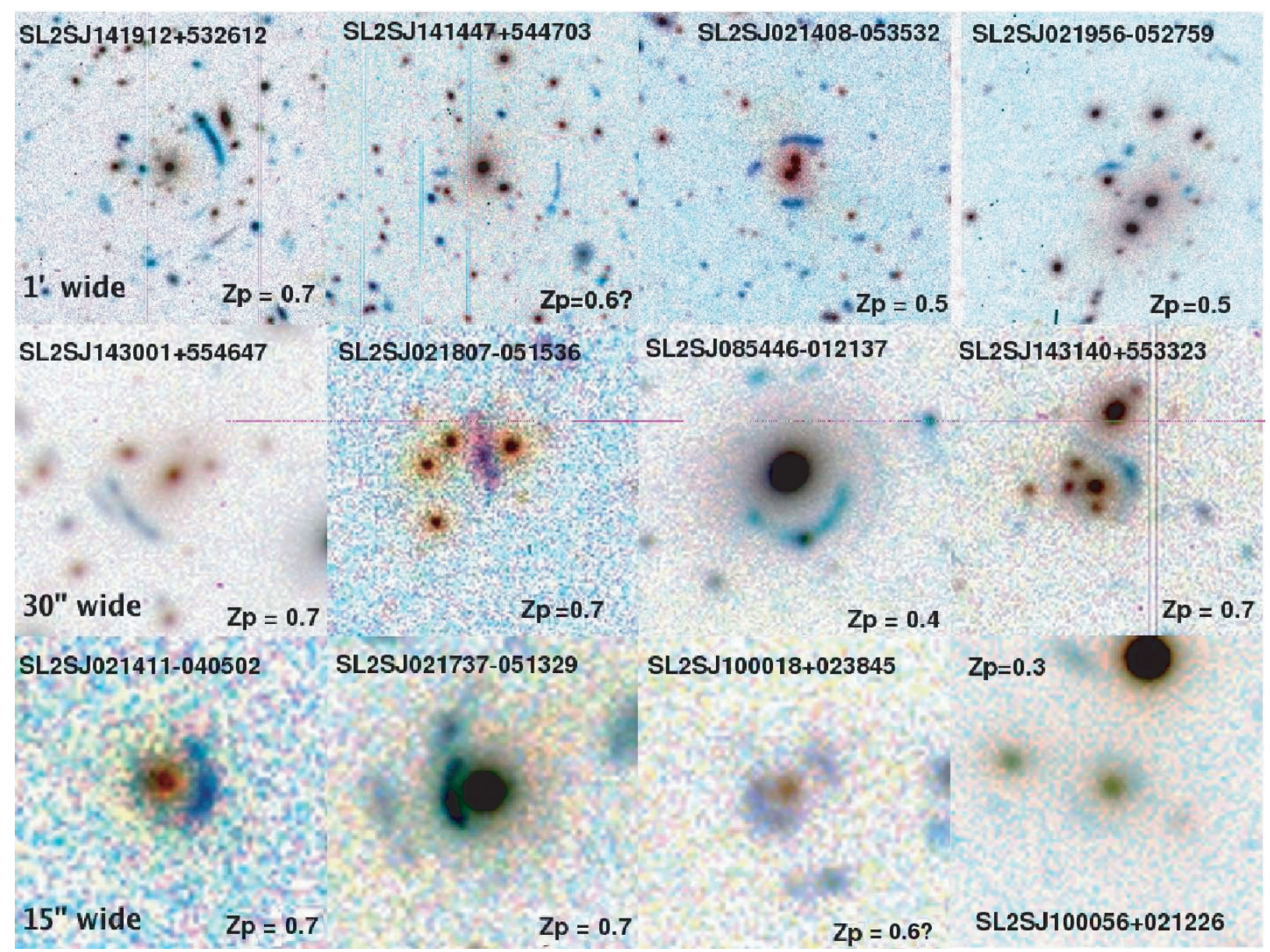

Fig. 2. RGB $\left(i^{\prime} g^{\prime} u^{*}\right)$ mosaic of SL2S candidates showing the three regimes probed by the sample. Top examples show $1^{\prime}$-wide vignettes of SL2S cluster lenses $\left(>10^{13} h^{-1} M_{\odot}\right)$. Middle examples show $30^{\prime \prime}$-vignettes of SL2S intermediate mass lenses (galaxy groups; $\sim 10^{13} h^{-1} M_{\odot}$ ). Bottom line shows $15^{\prime \prime}$-wide vignettes of Einstein rings around single galaxy lenses $\left(<10^{13} h^{-1} M_{\odot}\right)$. The estimated photometric redshift for the deflector is marked.

Keeton 2001; Warren \& Dye 2003; Brewer \& Lewis 2006). It is clearly beyond the scope of this paper to review them. Our team has developped many parametric and non-parametric codes, using a variety of optimization algorithms to model the full spectrum of the mass regimes, therefore mostly adapted to the CFHTLS-SL2S sample.

\section{Preliminary sample from the T0002 release}

\subsection{Description of the sample}

The preliminary analysis of the CFHTLS T0002 release led to the discovery of 43 candidate strong lenses listed in Table 2. Most of the CFHTLS-SL2S candidates that we present here were extracted using the arc detector, which turned out to be very efficient irrespective of the arc size or the lens environment. The list also contains 10 rings first identified in the COSMOS field and then recovered in the CFHTLS-D2 data. Hence, this preliminary sample is considered a feasability study for the SL2S project, demonstrating that a wide mass range will be uncovered by the CFHTLS-SL2S. This sample should not be used for quantitative statistical studies.

In the current selection process, the automated software extracts a list of candidates and creates color images of each of them for subsequent visual inspection. The SL2S database (http: //www. cfht . hawaii.edu/ cabanac/ $\mathrm{SL} 2 \mathrm{~S} /$ ) is presently under construction and includes for each system the magnitudes of the lens, its photometric redshift estimates, using either the latest version of the HyperZ code (Bolzonella et al. 2000), or LePhare, a code based on a Bayesian approach (Ilbert et al. 2006). A few geometrical properties for the lensed source (arc radius, magnitudes when known) are also included. Table 2 summarizes these measurements for our preliminary sample.

\subsection{Global properties of the sample}

Although this sample is not yet complete, we already see three classes, split according to their arc radius, hence to their mass regime. A detailed mass classification will only be possible when spectroscopic redshifts of the lenses and the sources are known, and when high-resolution imaging yields accurate lens modeling.

- The most conspicuous class includes giant arcs with radii $>7 "$. In the T0002 release sample, this class contains 4 candidates, detected over an effective area of 28 sq. degrees. By simple extrapolation, one can expect to find about 15-20 of these giant arcs in the complete CFHTLS $170 \mathrm{deg}^{2}$ (equivalent to about $100 \mathrm{deg}^{2}$ unmasked clear sky). The top line of Fig. 2 mosaic shows the 4 giant arcs with arc radii $>7 "$. Usually such features appear in massive clusters often associated with strong X-ray emission. 

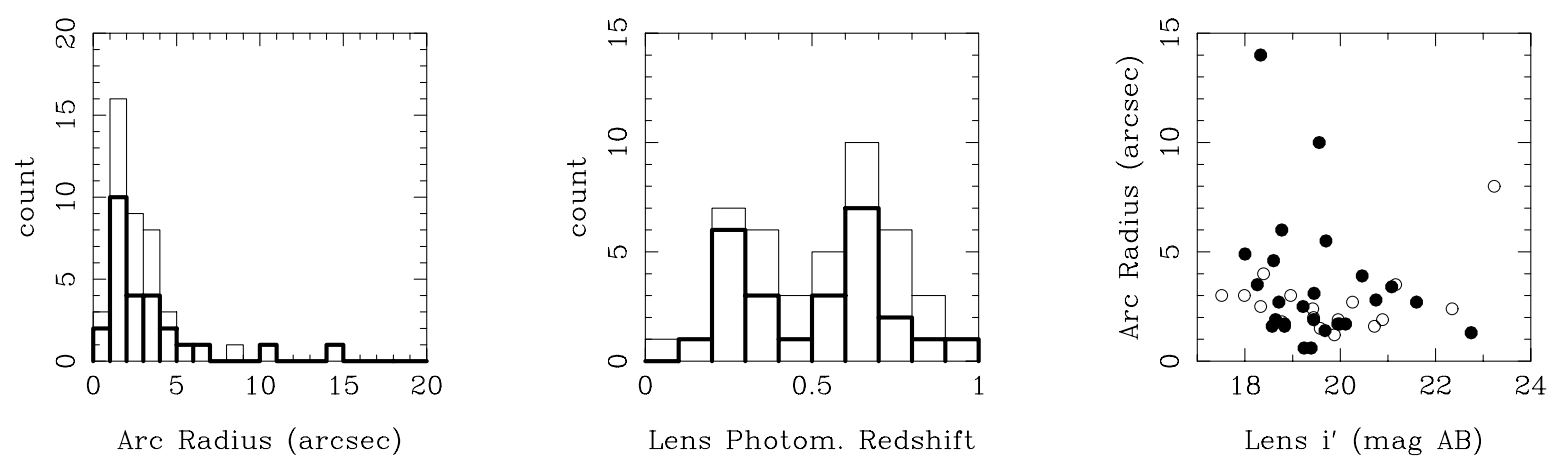

Fig. 3. T0002 release sample basic characteristics: Arc radius histogram (left), deflector/lens redshift histogram (middle). Arc radius versus central galaxy $i^{\prime}$ magnitude of the deflector/lens (right). Thick lines and filled circles correspond to quality 1 candidates. Thin lines and open circles correspond to quality 2 candidates (cf. text and Table 2 ).

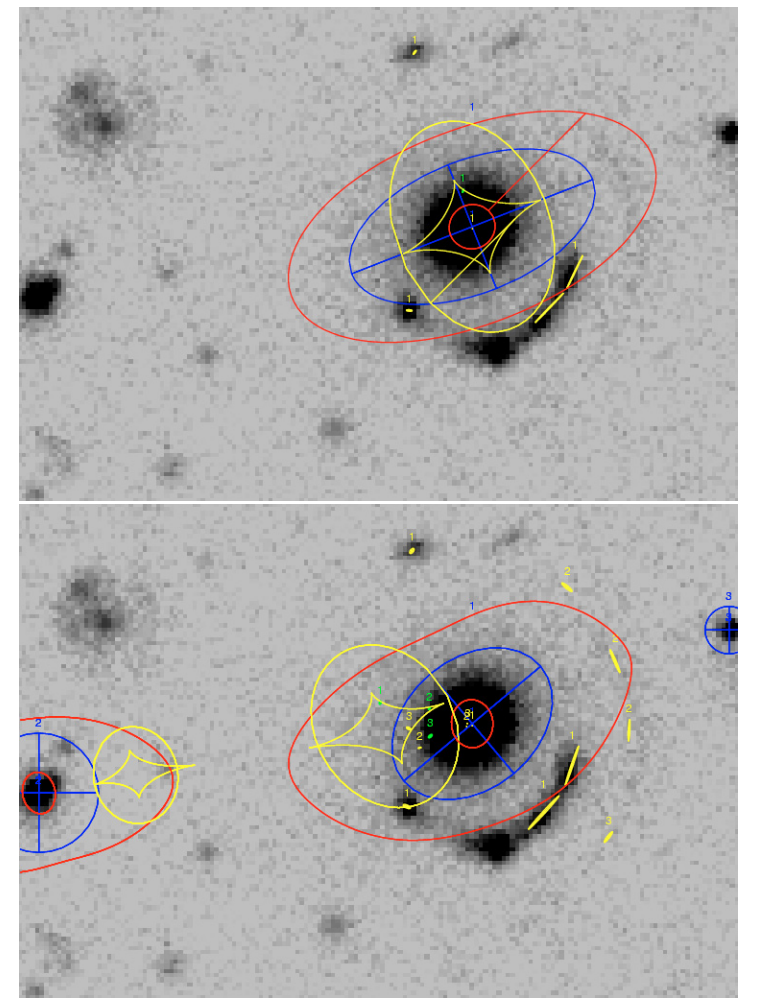

Fig. 4. Top: simple modelling of SL2SJ085446-012137 using LensTool (Kneib 1993, http://www . oamp.fr/cosmology/lenstool/) where only the central galaxy is used as a deflector. The source near the upper cusp of the radial critical line divides into 4 virtual images, three on the south half and one in the north. Bottom: same lens with a more complex modelling including surrounding galaxies as potential sources of shear. High-resolution imaging and surface brightness information will allow one to remove part of the degeneracy of lens models.

- The second class of lenses is mostly made of intermediate mass deflectors, showing arc radii in the range $3-7^{\prime \prime}$. We found 13 such candidates ( 8 additional candidates with radii $\sim 2.5^{\prime \prime}$ which might belong to small groups) in the CFHTLS T0002 release ( $\sim 75$ are expected over the complete survey). The middle line of Fig. 2 shows a selection of these intermediate mass candidates. They represent the largest sample of intermediate mass lens detected so far and the CFHTLS-SL2S seems to be particularly efficient at detecting this class of lenses. The lens modeling of groups might be more complex than the modeling of giant arcs in clusters of galaxies, often dominated by a bright central galaxy, or of rings around an isolated elliptical galaxy. As an illustration, Fig. 4 shows the possible influence of the external shear due to group members on the main arc modeling for the lens SL2SJ085446-012137. Only HST imaging will allow us to reduce these degeneracies.

- The third class is made up of compact ring candidates commonly associated with isolated galaxies, with ring radii $<3^{\prime \prime}$. This class is expected to be the most populated one. If we follow Oguri's predictions (2006) we expect the presence of about 100 rings in the T0002 Wide survey release by a simple scaling between the intermediate mass lenses and the galaxy mass lenses. If we exclude the 10 rings identified in the COSMOS field (D2) using HST imaging and detected a posteriori in the CFHTLS data, there are only 12 extra candidates detected in our sample. This shows that appropriate detection of ring candidates is not yet fully operational and requires more developments and tests, along the lines described in Sect. 3.2. The bottom line of Fig. 2 shows a selection of the few compact arc or ring candidates already identified.

The photometric redshift histograms of the deflectors of the total sample (thin line) and almost undisputable candidates (thick line) (Fig. 3) show a wide distribution up to $z \sim 1$. Ilbert et al. (2006) claim that LePhare Bayesian photometric redshifts are accurate to $\sigma_{\Delta z} \sim 0.05$, with only $4 \%$ of so-called catastrophic errors, i.e. when $\sigma_{\Delta z}>0.15$, for objects brighter than $i_{\mathrm{AB}}<24$, based on the 5 CFHTLS colors. However, photometric redshifts of almost all the lenses detected in the Wide survey are determined from 3 photometric colors only $\left(g^{\prime}, r^{\prime}, i^{\prime}\right)$ and should be taken with caution, as the expected rate of catastrophic errors increases to $\sim 20 \%$. The observed bimodal distribution with a peak about $z=0.3$ and another around $z=0.6$ could be a statistical fluctuation or a real effect due to the fact that for the limiting magnitude of the CFHTLS Wide survey the distribution of sources is expected to peak just above $z \sim 0.6$. A better determination of the photometric redshifts, including near-IR data, or a spectroscopic measurement through a dedicated spectroscopic follow-up are planned to derive the observed redshift distribution of the sample. With an average lens redshift $z \sim 0.5$ the SL2S will go beyond the SLACS and will provide a galaxy and group sample able to probe the evolution of mass at higher redshift. Figure 3 also shows the expected loose correlation between arc radius and lens apparent magnitude in $i^{\prime}$ band, suggesting that the CFHTLS is not strongly biased in any mass regime, but seems, on the contrary, to be sensitive to the complete parameter space. 


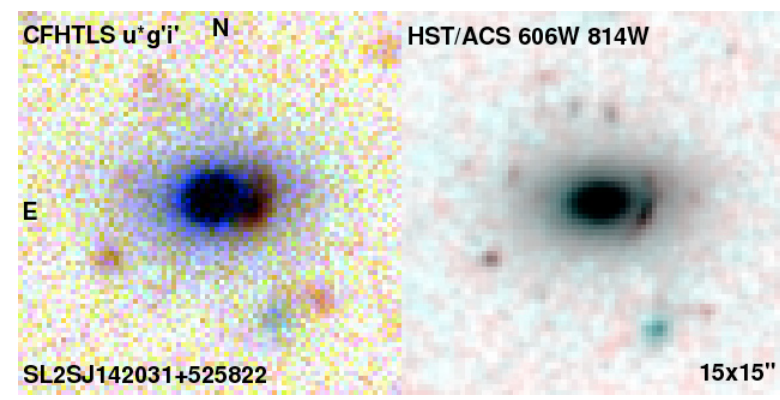

Fig. 5. Left: CFHTLS RGB $\left(i^{\prime} g^{\prime} u^{*}\right)$ image of the candidate SL2SJ142031+525822. Right: HST RGB (814W-606W-600W) image of the same field clearly showing a bright arc akin to lensing distortion but a spectrum is needed to confirm that the arc is indeed at a higher redshift and not a star-forming spiral arm or a merging galaxy.

\subsection{Notes on selected candidates}

SL2SJ021408-053532: A bright system of fold arcs around a compact group of three galaxies at $z_{\text {phot }} \sim 0.5$ (Fig. 2), absolute $B$ magnitude $M_{B}=-22.4$, and a homogeneous population of galaxies with similar color within $2^{\prime}$.

SL2SJ021411-040502: Typical example of a compact ring candidate around a galaxy in a loose group or cluster at $z_{\text {phot }} \sim$ 0.7. These cases seem to be common in the CFHTLS and might provide interesting constraints on the sub-structure of DM haloes.

SL2SJ100013+022249: This is an example of a compact lensing candidate which does not appear very clearly in CFHTLS imaging, but stands out in the HST cosmos field, and the CFHT normalized $g^{\prime}-i^{\prime}$ image (Fig. 1).

SL2SJ141912+532612: This massive cluster showing multiple arcs is not a new detection. Gladders et al. (2003) already discovered the same lensing cluster called RCS1419.2+5326 within the Red Cluster Sequence survey. The photometric redshift of SL2SJ141912+532612 is $z_{l}=0.65$ based on 3 colors only $\left(g^{\prime} r^{\prime} i^{\prime}\right)$ but is remarkably similar to the spectroscopic redshift of RCS1419.2+5326 $z_{\text {spectro }}=0.64$. HST imaging in F814W is available; HST proposal 10626, PI Loh. This system shows at least three arcs at 10, 14.5, and 17" (Fig. 2).

SL2SJ142031+525822: Example of a relatively compact arc/ring candidate at $z_{\text {phot }} \sim 0.3$ (and likely higher), the confirmation of which typically requires a spectroscopic follow-up in addition to higher-resolution imaging. This candidate fortunately falls within the Groth Strip Survey and has already been imaged with HST/ACS in F606W and F814W (Simard et al. 2002; Vogt et al. 2005). Figure 5 shows $1 \times 1^{\prime}$ RGB images of the CFHTLS $\left(i^{\prime} g^{\prime} u^{*}\right)$ and HST (814W-606W-600W). This candidate is particularly interesting for the large ellipticity the deflector, a rare occurence among isolated strong lenses.

SL2SJ142209+524652: This candidate is a very extended arc surrounding a compact group of two galaxies at $z_{\text {phot }} \sim 0.2$ and offers a very unorthodox lensing configuration.
SL2SJ143140+553323 Example of lensing by two groups occupying the same line of sight at $z_{\text {phot }} \sim 0.5-0.6$, and having a discernable impact on the lensing distortion with a fold arc and a potential saddle pair.

\section{Discussion}

\subsection{Dark matter distribution in galaxy groups}

Only a handful of galaxy groups associated with strong lenses are known, mostly as an environmental association with a galaxy lens (Grant et al. 2004; Fassnacht et al. 2005; Auger et al. 2006). In such cases the group reveals its presence because it produces an external shear acting as a perturbation in the lens modeling. But so far almost no lenses with image splitting between 3 and 7 arcsec have been observed corresponding to halo masses of $M_{\text {halo }} \gtrsim 10^{13} h^{-1} M_{\odot}$, except the "historical" double quasar Q $0957+561$ (Walsh et al. 1979); this system is centered in a giant elliptical galaxy embedded in a poor cluster of galaxies at redshift 0.36 (Angonin-Willaime et al. 1994), and recently the 2 lensed quasars of the SDSS (Oguri et al. 2005).

There are several important reasons to study groups. $60 \%$ of the galaxies belong to groups which play a key role in the assembly of structures. Merging processes in groups can have a strong effect on the star formation rate and probably on the growth of the super massive black hole that lies in their dominant ellipticals. Much work has been done in recent years using large surveys of groups in the SDSS spectroscopic data base (Weinmann et al. 2006), within the DEEP survey (Coil et al. 2006) and/or with X-ray surveys (Miles et al. 2004, GEMS survey). These first results did not succeed in giving a definitive picture of the average group mass profile, the relation between the light and total mass distribution, or the relation between the different galaxy type fractions (segregation effect as observed in clusters).

Part of the problem lies in the fact that sub-structures of simulated DM haloes can be very complex and the definition of a DM halo center is scale dependent. Strong lensing arcs in the center of group haloes can provide a unique measure of the total projected density profile, where dynamical estimates only provide constraints on the baryonic mass with the strong assumption that groups are virialized systems.

Recent weak shear analyses have been done by roughly stacking two classes of groups detected in the CNOC2 spectroscopic galaxy survey in order to derive structural and $M / L$ properties (Möller et al. 2002; Parker et al. 2005). They found that very poor groups with a mean velocity dispersion of $200 \mathrm{~km} \mathrm{~s}^{-1}$ have an average Einstein radius of about $1^{\prime \prime}$ (very similar to single massive ellipticals) while the most massive groups with a velocity dispersion close to $300 \mathrm{~km} \mathrm{~s}^{-1}$ have properties more similar to clusters. It also appears that a total mass of $10^{13} \mathrm{~h}^{-1} M_{\odot}$ might correspond to a transition mass scale for the $M / L$ ratio and the related star formation rate.

$\mathrm{X}$-ray observations have also revealed that there is a peculiar class of old groups dominated by a bright elliptical galaxy that probably formed at an early time in the universe (D'Onghia et al. 2005; Ulmer et al. 2005). Likewise, Guimarães et al. (2005) used the QSO magnification bias in the $2 \mathrm{dF}$ Galaxy Redshift Survey to measure a surprisingly high lensing signal, suggesting that some groups are more massive than expected.

The SL2S group sample is well adapted to address these questions. 


\subsection{Searching a large sample of gravitational rings}

Based on our simple extrapolations, CFHTLS Einstein rings will clearly outnumber all the already existing galaxy lens samples. Once fully optimised, the SL2S ring detection procedure will provide ca. 400 rings in the $170 \mathrm{deg}^{2}$ of the CFHTLS wide survey. Even if we restrict ourselves to a more conservative number of $\sim 300$ robust SL2S systems, confirmed with HST imaging, it will increase the present known sample by a factor of 3 over a large redshift range $0.3-1$. Thus a comprehensive statistical analysis of this class of lenses will be possible, looking for variations as a function of cosmic time and galaxy luminosity, type and environment, and extending the results of the SLACS on the evolution of the E/S0 fundamental plane and the M/L ratio of normal galaxies, following their pionneering method of combined spectroscopy-based dynamical analysis and lens modelling.

\section{Conclusion}

We have presented the guidelines of the CFHTLS Strong Lensing Legacy Survey, a survey aimed at detecting all the lensed features in the CFHTLS data for the study of the dark matter halo distributions from isolated galaxies to groups and large clusters up to a redshift of 1 . A series of three automated softwares are being developed: an arc detector mainly focused on giant arcs and arclets, a ring detector for the detection of compact Einstein rings and a multiplet detector optimized in multiplet images showing generic lensing properties irrespective of the deflector's light. The preliminary sample is based on the CFHTLS T0002 release and led to the discovery of ca. 40 candidates spanning the complete mass range (the current release, T0003, increases the sample by $50 \%$. The best candidates will be followed up by HST in Cycle 15, snapshot program 10876). An unexpectedly large number of intermediate mass deflectors akin to galaxy groups is present in this sample. We therefore expect to address many questions related to galaxy group physics through the project, like the mass profile transition between cluster-like NFW and galaxy-like isothermal profiles, dark matter substructures in intermediate mass haloes, star formation processes and their feedback in the inter-galactic medium, and stellar mass assembly with redshift. Moreover, although the detection procedure is not yet fully optimised, we expect to uncover and study the most abundant population of rings, known to be present in the CFHTLS images. This will allow us to extend the work of the SLACS team to higher redshifts for a better undertanding of the dark matter distribution and impact of baryon cooling in galaxy haloes, and the evolution of the Fundamental Plane and mass assembly of isolated galaxies.

By the end of the survey (2008), we expect to build a sample of about 500 lenses with well-controlled selection effects. This will allow us to investigate the probability distribution of lens splitting angles across the whole lens mass range from galaxies to clusters. Following the pioneering work of Kochanek \& White (2001), the studies by Ofek et al. (2003) and more recently Oguri (2006) demonstrate how crucial it is to have a large observational sample to be able to make meaningful comparisons with simulations. The CFHTLS-SL2S is likely to become the most complete survey of strong lenses available for many years.

Acknowledgements. The SL2S collaboration would like to thank P. Petitjean for sharing his MegaCam dataset prior to publication, and Cécile Faure and the COSMOS team for sharing their Einstein ring candidates in CFHTLS D2. This work also uses Groth Strip Survey data obtained or processed with support of the National Science Foundation grants AST 95-29028 and AST 00-71198.

\section{References}

Alard, C. 2006, [arXiv:astro-ph/0606757]

Angonin-Willaime, M.-C., Soucail, G., \& Vanderriest, C. 1994, A\&A, 291, 411 Auger, M. W., Fassnacht, C. D., Abrahamse, A. L., Lubin, L. M., \& Squires, G. K. 2006, [arXiv: astro-ph/0603448]

Blandford, R. D., \& Narayan, R. 1992, ARA\&A, 30, 311

Bolton, A. S., Burles, S., Koopmans, L. V. E., Treu, T., \& Moustakas, L. A. 2006, ApJ, 638, 703

Bolzonella, M., Miralles, J.-M., \& Pelló, R. 2000, A\&A, 363, 476

Brewer, B. J., \& Lewis, G. F. 2006, ApJ, 637, 608

Broadhurst, T., Benítez, N., Coe, D., et al. 2005, ApJ, 621, 53

Cabanac, R. A., Valls-Gabaud, D., Jaunsen, A. O., Lidman, C., \& Jerjen, H. 2005, A\&A, 436, L21

Coil, A. L., Gerke, B. F., Newman, J. A., et al. 2006, ApJ, 638, 668

D’Onghia, E., Sommer-Larsen, J., Romeo, A. D., et al. 2005, ApJ, 630, L109

Fassnacht, C., Lubin, L., McKean, J., et al. 2005, in IAU Symp., ed. Y. Mellier, \& G. Meylan, 311

Gavazzi, R., Fort, B., Mellier, Y., Pelló, R., \& Dantel-Fort, M. 2003, A\&A, 403, 11

Gladders, M. D., Hoekstra, H., Yee, H. K. C., Hall, P. B., \& Barrientos, L. F. 2003, ApJ, 593, 48

Grant, C. E., Bautz, M. W., Chartas, G., \& Garmire, G. P. 2004, ApJ, 610, 686 Guimarães, A. C. C., Myers, A. D., \& Shanks, T. 2005, MNRAS, 362, 657

Hall, P. B., Yee, H. K. C., Lin, H., et al. 2000, AJ, 120, 1660

Hetterscheidt, M., Erben, T., Schneider, P., et al. 2005, A\&A, 442, 43

Hewett, P. C., Warren, S. J., Willis, J. P., Bland-Hawthorn, J., \& Lewis, G. F.

2000, in Imaging the Universe in Three Dimensions, ed. W. van Breugel, \&

J. Bland-Hawthorn, ASP Conf. Ser., 195, 94

Hoekstra, H., Yee, H. K. C., \& Gladders, M. D. 2004, ApJ, 606, 67

Horesh, A., Ofek, E. O., Maoz, D., et al. 2005, ApJ, 633, 768

Ilbert, O., Arnouts, S., McCracken, H. J., et al. 2006,

[arXiv: astro-ph/0603217]

Keeton, C. R. 2001, ApJ, submitted [arXiv: astro-ph/0102340]

Kneib, J. P., Mellier, Y., Fort, B., \& Mathez, G. 1993, A\&A, 273, 367

Kneib, J.-P., Hudelot, P., Ellis, R. S., et al. 2003, ApJ, 598, 804

Kochanek, C. S., \& White, M. 2001, ApJ, 559, 531

Kochanek, C. S., Falco, E. E., Impey, C. D., et al. 2000, ApJ, 543, 131

Kochanek, C. S., Keeton, C. R., \& McLeod, B. A. 2001, ApJ, 547, 50

Koopmans, L. V. E., Treu, T., Bolton, A. S., Burles, S., \& Moustakas, L. A. 2006, [arXiv:astro-ph/0601628]

Lenzen, F., Schindler, S., \& Scherzer, O. 2004, A\&A, 416, 391

Mathews, W. G., Faltenbacher, A., Brighenti, F., \& Buote, D. A. 2005, ApJ, 634, L137

Mellier, Y. 2002, Space Sci. Rev., 100, 73

Mellier, Y., \& Meylan, G., eds. 2005, Gravitational Lensing Impact on Cosmology (S225)

Miles, T. A., Raychaudhury, S., Forbes, D. A., et al. 2004, MNRAS, 355, 785

Miralda-Escude, J. 1995, ApJ, 438, 514

Miralda-Escude, J., \& Lehar, J. 1992, MNRAS, 259, 31P

Möller, O., Natarajan, P., Kneib, J.-P., \& Blain, A. W. 2002, ApJ, 573, 562

Ofek, E. O., Rix, H.-W., \& Maoz, D. 2003, MNRAS, 343, 639

Oguri, M. 2006, MNRAS, 367, 1241

Oguri, M., Inada, N., Hennawi, J. F., et al. 2005, ApJ, 622, 106

Parker, L. C., Hudson, M. J., Carlberg, R. G., \& Hoekstra, H. 2005, ApJ, 634, 806

Rusin, D., \& Kochanek, C. S. 2005, ApJ, 623, 666

Rusin, D., Kochanek, C. S., Falco, E. E., et al. 2003, ApJ, 587, 143

Saha, P., \& Williams, L. L. R. 1997, MNRAS, 292, 148

Schneider, P., Ehlers, J., \& Falco, E. E. 1992, Gravitational Lenses XIV (Berlin,

Heidelberg, New York: Springer-Verlag), also Astronomy and Astrophysics Library

Seidel, G., \& Bartelmann, M. 2006, [arXiv:astro-ph/0607547]

Seljak, U., Makarov, A., Mandelbaum, R., et al. 2005, Phys. Rev. D, 71, 043511

Simard, L., Willmer, C. N. A., Vogt, N. P., et al. 2002, ApJS, 142, 1

Treu, T., Ellis, R. S., Liao, T. X., \& van Dokkum, P. G. 2005, ApJ, 622, L5

Ulmer, M. P., Adami, C., Covone, G., et al. 2005, ApJ, 624, 124

Vogt, N. P., Koo, D. C., Phillips, A. C., et al. 2005, ApJS, 159, 41

Walsh, D., Carswell, R. F., \& Weymann, R. J. 1979, Nature, 279, 381

Warren, S. J., \& Dye, S. 2003, ApJ, 590, 673

Warren, S. J., Hewett, P. C., Lewis, G. F., et al. 1996, MNRAS, 278, 139

Weinmann, S. M., van den Bosch, F. C., Yang, X., \& Mo, H. J. 2006, MNRAS, 366,2

Willis, J. P., Hewett, P. C., Warren, S. J., Dye, S., \& Maddox, N. 2006, MNRAS, 369,1521 